\section{A posteriori teleportation}

Samuel L. Braunstein* and H. J. Kimble ${ }^{\dagger}$

*SEECS, University of Wales, Bangor LL57 1UT, UK

${ }^{\dagger}$ Norman Bridge Laboratory of Physics 12-33,

California Institute of Technology, Pasadena, CA 91125

The article by Bouwmeester et al $\mathbf{1}$ on experimental quantum teleportation constitutes an important advance in the burgeoning field of quantum information. The experiment was motivated by the proposal of Bennett et all $\mathrm{B}$ in which an unknown quantum state is 'teleported' by Alice to Bob. As illustrated in Fig. 1, in the implementation of this procedure, by Bouwmeester et al., an input quantum state is 'disembodied' into quantum and classical components, as in the original protocol.2 However, in contrast to the original scheme, Bouwmeester et al.'s procedure necessarily destroys the state at Bob's receiving terminal, so a 'teleported' state can never emerge as a freely propagating state for subsequent examination or exploitation. In fact, teleportation is achieved only as a postdiction.

Bouwmeester et al. used parametric down-conversion from two sources (SI, SII in Fig. 1) in an attempt to teleport the polarization state of a single-photon wavepacket (in beam 1) from Alice's sending station to Bob's receiving station (in beam 3). Statistics consistent with teleportation are obtained for events with a fourfold coincidence (from detectors $\mathrm{f} 1$ and $\mathrm{f} 2, \mathrm{~d} 1$ or $\mathrm{d} 2$, and $\mathrm{p}$ ). We ask whether the detection of all four quanta is essential for teleportation in this scheme. To answer this question, we calculated the teleportation fidelity, $F$, when the coincidence condition is relaxed to exclude detection at Bob's station (d1, d2).

Under relaxed conditions, requiring only threefold coincidence of detectors $\mathrm{p}$ and (f1, f2), teleportation is achieved when the fields of beams 1 and 3 match with sufficiently high fidelity. In the simplest approximation, type II parametric down-conversion on modes $(i, j)$ generates wavepacket states as follows:

$$
A_{0}|0\rangle_{i j}+A_{1}\left|\psi^{-}\right\rangle_{i j}+A_{2}|\chi\rangle_{i j}+\cdots,
$$

where $A_{0}, A_{1}$ and $A_{2}$ are the coefficients for obtaining no (vacuum), one and two down-converted pairs, respectively, and $(i, j)=(1,4),(2,3)$. Of these terms, only states corresponding to the second term are selected by fourfold coincidence, as specified by equations (2) and (3) of ref. 1. However, anything less than complete destruction of the output 3 necessarily leaves undesirable terms that reduce $F$.

The initial input state to Alice's station, $|\Phi\rangle$, is prepared by detecting the state in field 4 at detector $\mathrm{p}$, which projects the field in beam 1 accordingly. Joint detection at $(f 1, f 2)$ then provides threefold coincidence with $\mathrm{p}$, yielding a statistical mixture for the field 3 arriving at Bob's station. The fraction of the state $|\Phi\rangle$ in this mixture gives $F$. To lowest order in the down-converter cou-

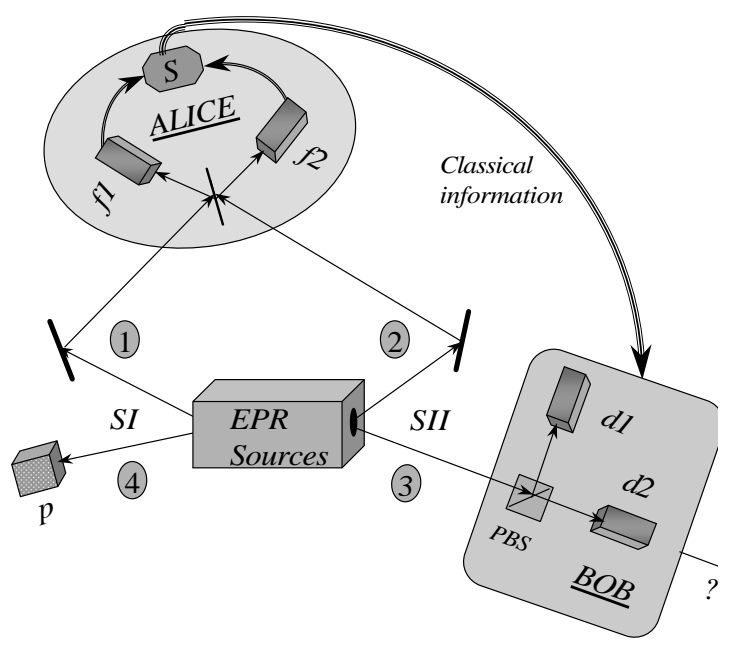

FIG. 1. The teleportation set-up of ref. 1). PBS, polarizing beam splitter.

pling strength, the Bouwmeester et al. scheme yields a 50:50 mixture of the vacuum state $|0\rangle$ and the desired state $|\Phi\rangle$, with $F=1 / 2$, so that there is never a physical state with high teleportation fidelity. Indeed, Bob could achieve this same fidelity, $F=1 / 2$, by abandoning teleportation altogether and transmitting randomly selected polarization states. Faced with this state of affairs, the experiment of ref. 1 obtains a surrogate for high fidelity by destructively recording the field 3 at (d1, d2).

We emphasize that the nature of the mixture containing the vacuum state has definite physical implications, which can be verified by more general measurements than photon counting (for example, by quantum-state tomography). Moreover, the freedom of a potential consumer of the output from Bob's receiving station to select alternative detection strategies means that classical analogies fail.

To achieve conventional a priori teleportation, the setup in ref. 1 would have to be modified to eliminate the vacuum from the mixture. Because the vacuum appears when two pairs of $(1,4)$ photons are created, we might seek to resolve one- and two-photon detection events at p. Upgraded detection (for example, by cascading conventional detectors) could provide an effective remedy. Appropriate selection could be implemented with a polarization-independent quantum non-demolition measurement of the total photon number at Bob's end. Alternatively, pre-selection could be implemented by enhancing the coupling between modes $(2,3)$ relative to modes $(1,4)$.

Despite our comments, the experiment of Bouwmeester et al. is a significant achievement in demonstrating the non-local structure of teleportation.

\footnotetext{
${ }^{1}$ Bouwmeester, D. et al. Nature 390, 575-579 (1997).

2 Bennett, C. H. et al. Phys. Rev. Lett. 70, 1895-1899 (1993).
} 